\title{
Next to subleading soft-graviton theorem in arbitrary dimensions
}

\section{Chrysostomos Kalousios ${ }^{a}$ and Francisco Rojas $^{b}$}

${ }^{a}$ ICTP South American Institute for Fundamental Research, Instituto de Física Teórica, UNESP-Universidade Estadual Paulista, R. Dr. Bento T. Ferraz 271, Bl. II, 01140-070, São Paulo, SP, Brasil

${ }^{b}$ Instituto de Física Teórica, UNESP-Universidade Estadual Paulista, R. Dr. Bento T. Ferraz 271, Bl. II, 01140-070, São Paulo, SP, Brasil

E-mail: ckalousi@ift.unesp.br, frojasf@ift.unesp.br

ABSTRACT: We study the soft graviton theorem recently proposed by Cachazo and Strominger. We employ the Cachazo, He and Yuan formalism to show that the next to subleading order soft factor for gravity is universal at tree level in arbitrary dimensions.

Keywords: Scattering Amplitudes, Field Theories in Higher Dimensions

ARXiv EPRINT: 1407.5982 


\section{Contents}

1 Introduction 1

2 Review and setup of the problem 3

3 Evaluation of $M_{n}^{(2)} \quad \mathbf{5}$

3.1 Evaluation of $m_{1}$

3.2 Evaluation of $m_{2} \quad 6$

3.3 Evaluation of $m_{3} \quad 8$

4 Action of $S^{(2)}$ on the amplitude $\quad 10$

4.1 Evaluation of $s_{1} \quad 11$

$\begin{array}{lll}4.2 & \text { Evaluation of } s_{2} \text { and } s_{3} & 12\end{array}$

$\begin{array}{lll}4.3 & \text { Evaluation of } s_{4} & 12\end{array}$

\section{Introduction}

The study of the soft graviton amplitudes dates back to Weinberg [1, 2] where the leading soft behavior was obtained. In [3-5] a new soft graviton theorem, conjectured to be the Ward identities of a new symmetry of the quantum gravity S-matrix, ${ }^{1}$ was proposed. Cachazo and Strominger [8] have recently shown that the new conjectured soft behavior, through subleading and next-to-subleading orders in the soft expansion, has a universal form in four spacetime dimensions at tree level. ${ }^{2}$ An extension to gluons for the first subleading soft behavior at tree level was reported in [15] and in [16] it was demonstrated that the conformal invariance of tree level gauge theory amplitudes in four spacetime dimensions determines the form of the first subleading theorem.

Very recently it has been shown that the form of the subleading and next-to-subleading terms in the soft expansion in $D$ dimensions - for both gauge theory and gravity is greatly constrained by the requirements from Poincaré symmetry, gauge invariance, and a self consistency condition originating from the distributional nature of scattering amplitudes [17]. Moreover, the subleading and next-to-subleading terms were determined up to a numerical constant for each external leg. ${ }^{3}$ Using Feynman diagram techniques the first subleading theorem for soft gluons and gravitons was also confirmed in [18]. The

\footnotetext{
${ }^{1}$ This new proposed symmetry is an extension of the Bondi, van der Burg, Metzner and Sachs (BMS) symmetry $[6,7]$.

${ }^{2}$ Early results for soft photons at subleading order were obtained in [9-12]. Gross and Jackiw, using dispersion relation methods, derived the subleading soft factor for graviton scattering off scalars in [13], and White revised the subject in [14] using path integral resummation techniques.

${ }^{3}$ However, the authors also point out that these numerical factors are completely fixed when specializing to four spacetime dimensions.
} 
authors of [19] have shown that on-shell gauge invariance determines the complete form of the first subleading soft theorem in gauge theory and the first two subleading soft theorems for gravity. Using the Cachazo, He, Yuan (CHY) formula [20], the tree level universality of the soft behavior to first subleading order has been shown to hold in $D$ dimensions $[21,22]$. The purpose of the present note is to use the CHY formula to prove the universal nature of the next-to-subleading soft graviton theorem at tree level in arbitrary dimensions.

Studies on loop corrections to subleading soft theorems have been presented in [23-25]. Progress in the context of string theory has been reported in [26, 27] and also in [28, 29] relevant for recent twistor constructions. More recent progress on soft theorems in the context of massless QED has appeared in [30, 31].

The conjecture of [8] states, for an on-shell tree level $n$-graviton amplitude $M_{n}$, that

$$
M_{n}=\left(\frac{1}{\lambda} S^{(0)}+S^{(1)}+\lambda S^{(2)}+\mathcal{O}\left(\lambda^{2}\right)\right) M_{n-1},
$$

where $n$ is taken to be the soft particle with momentum $k_{n}$ and we scale the momentum $k_{n} \rightarrow \lambda k_{n}$ and take the limit when $\lambda$ approaches zero. In the above,

$$
S^{(0)}=\sum_{a=1}^{n-1} \frac{\epsilon_{\mu \nu} k_{a}^{\mu} k_{a}^{\nu}}{k_{n} \cdot k_{a}}
$$

is Weinberg's soft theorem with $\epsilon_{\mu \nu}$ denoting the polarization tensor of the soft graviton and the gravitational constant has been set to 1 . The conjectured forms of the subleading and next-to-subleading theorems are

$$
S^{(1)}=-i \sum_{a=1}^{n-1} \frac{\epsilon_{\mu \nu} k_{a}^{\mu} k_{n \lambda} J_{a}^{\lambda \nu}}{k_{n} \cdot k_{a}}, \quad S^{(2)}=-\frac{1}{2} \sum_{a=1}^{n-1} \frac{\epsilon_{\mu \nu} k_{n \rho} J_{a}^{\rho \mu} k_{n \lambda} J_{a}^{\lambda \nu}}{k_{n} \cdot k_{a}} .
$$

In order to treat gluon and graviton polarizations on an equal footing one can choose to write the graviton polarization for the $a^{\text {th }}$ particle as

$$
\epsilon_{a \mu \nu}=\epsilon_{a \mu} \epsilon_{a \nu}
$$

where $a=1, \ldots, n-1$. Tracelessness and orthogonality to $k_{a}$ translate into $\epsilon_{a} \cdot \epsilon_{a}=0$ and $\epsilon_{a} \cdot k_{a}=0$ respectively. ${ }^{4}$

The subleading contributions to the soft theorem depend on the total angular momentum operator, which is ${ }^{5}$

$$
J_{a}^{\mu \nu}=i\left(k_{a}^{[\mu} \frac{\partial}{\partial k_{a \nu]}}+\epsilon_{a}^{[\mu} \frac{\partial}{\partial \epsilon_{a \nu]}}\right)
$$

for the $a^{\text {th }}$ particle. Note that in using this formula one should consider the polarization vectors $\epsilon_{a}^{\mu}$ to be independent of the momenta $k_{a}^{\mu}$.

This paper is organized as follows. In section 2 we review the CHY formalism [20] for tree level graviton amplitudes which is valid in arbitrary dimensions and, in this language,

\footnotetext{
${ }^{4}$ We do not use any other gauge condition in this work.

${ }^{5}$ We follow the convention $A_{(\mu} B_{\nu)}=A_{\mu} B_{\nu}+A_{\nu} B_{\mu}$ and $A_{[\mu} B_{\nu]}=A_{\mu} B_{\nu}-A_{\nu} B_{\mu}$.
} 
we set up the computation for the expansion of the amplitude up to next-to-subleading order in the soft parameter. We finish this section by stating the new soft theorem extended to $D$ dimensions. In section 3 we explicitly evaluate the tree level $n$-graviton amplitude at next-to-subleading order in the soft expansion. In section 4 we compute the action of the conjectured $S^{(2)}$ operator (1.3) onto the $(n-1)$-graviton amplitude, as stated in (1.1), and show that it perfectly matches with the next-to-subleading amplitude $M^{(2)}$ of section 3 , thus proving the theorem.

\section{Review and setup of the problem}

In this section we briefly review the CHY construction [20] for tree level graviton amplitudes. A key object is the scattering equations

$$
\sum_{b \neq a}^{n} \frac{k_{a} \cdot k_{b}}{\sigma_{a b}}=0, \quad a, b=1, \ldots, n
$$

with $\sigma_{a b} \equiv \sigma_{a}-\sigma_{b}$, where the $\sigma_{a}$ are in general complex valued quantities. Due to the $\mathrm{SL}(2, \mathbb{C})$ symmetry of $(2.1)$, these constitute a system of $n-3$ independent equations for the set $\left\{\sigma_{a}\right\}$ and one can arbitrarily fix three of the $\sigma_{a}$ variables. We will call $\sigma_{i}, \sigma_{j}, \sigma_{k}$ the three fixed $\sigma$ s. The gauge fixed amplitude is

$$
M_{n}=\int[d \sigma]_{n-4} d \sigma_{n} \prod_{a \neq i, j, k}^{n} \delta\left(f_{a}^{n}\right) E_{n}
$$

where we have employed the useful short notation

$$
f_{a}^{n} \equiv \sum_{b \neq a}^{n} \frac{k_{a} \cdot k_{b}}{\sigma_{a b}}, \quad[d \sigma]_{n-4} \equiv\left(\sigma_{p q} \sigma_{q r} \sigma_{r p}\right)\left(\sigma_{i j} \sigma_{j k} \sigma_{k i}\right) \prod_{c \neq p, q, r}^{n-1} d \sigma_{c} .
$$

In the above, $E_{n}$ is defined to be

$$
E_{n}=4 \operatorname{det}\left(\Psi_{x y}^{x y}\right) / \sigma_{x y}^{2}
$$

where $\Psi_{x y z^{\prime} \ldots}^{x y z \ldots}$ is obtained from the $2 n \times 2 n$ antisymmetric matrix $\Psi$ after removing rows $x, y, z, \ldots$ and columns $x, y, z^{\prime}, \ldots$ with $1 \leq x<y \leq n$. The explicit expression of $\Psi$ is given by

$$
\Psi=\left(\begin{array}{cc}
A & -C^{\mathrm{T}} \\
C & B
\end{array}\right)
$$

with the $n \times n$ matrices $A, B, C$ given by

$$
A_{a b}=\frac{k_{a} \cdot k_{b}}{\sigma_{a b}} \delta_{a \neq b}, \quad B_{a b}=\frac{\epsilon_{a} \cdot \epsilon_{b}}{\sigma_{a b}} \delta_{a \neq b}, \quad C_{a b}=\frac{\epsilon_{a} \cdot k_{b}}{\sigma_{a b}} \delta_{a \neq b}-\delta_{a b} \sum_{c \neq a}^{n} \frac{\epsilon_{a} \cdot k_{c}}{\sigma_{a c}},
$$

where we use $\delta_{a \neq b} \equiv 1-\delta_{a b}$ in order to avoid cluttering our equations. In [20] it was shown that the quantity $E_{n}$ is independent of the choice of $x$ and $y$. 
In order to expand the delta function appearing in (2.2) in powers of $\lambda$ we separate it into two parts

$$
\begin{aligned}
\prod_{a \neq i, j, k}^{n} \delta\left(f_{a}^{n}\right) & =\frac{1}{\lambda} \delta\left(\sum_{b=1}^{n-1} \frac{k_{n} \cdot k_{b}}{\sigma_{n b}}\right) \prod_{a \neq i, j, k}^{n-1} \delta\left(\sum_{b \neq a}^{n-1} \frac{k_{a} \cdot k_{b}}{\sigma_{a b}}+\lambda \frac{k_{a} \cdot k_{n}}{\sigma_{a n}}\right) \\
& =\delta\left(f_{n}^{n-1}\right)\left(\frac{1}{\lambda} \delta^{(0)}+\delta^{(1)}+\lambda \delta^{(2)}\right)+\mathcal{O}\left(\lambda^{2}\right),
\end{aligned}
$$

where we define

$$
\begin{aligned}
\delta^{(0)}= & \prod_{a \neq i, j, k}^{n-1} \delta\left(f_{a}^{n-1}\right), \quad \delta^{(1)}=\sum_{l \neq i, j, k}^{n-1} \frac{k_{l} \cdot k_{n}}{\sigma_{l n}} \delta^{\prime}\left(f_{l}^{n-1}\right)\left[\prod_{a \neq i, j, k, l}^{n-1} \delta\left(f_{a}^{n-1}\right)\right], \\
\delta^{(2)}= & \frac{1}{2} \sum_{l \neq i, j, k}^{n-1} \frac{k_{l} \cdot k_{n}}{\sigma_{l n}} \delta^{\prime}\left(f_{l}^{n-1}\right) \sum_{m \neq i, j, k, l}^{n-1}\left[\frac{k_{m} \cdot k_{n}}{\sigma_{m n}} \delta^{\prime}\left(f_{m}^{n-1}\right) \prod_{b \neq i, j, k, l, m}^{n-1} \delta\left(f_{b}^{n-1}\right)\right] \\
& +\frac{1}{2} \sum_{l \neq i, j, k}^{n-1}\left(\frac{k_{l} \cdot k_{n}}{\sigma_{l n}}\right)^{2} \delta^{\prime \prime}\left(f_{l}^{n-1}\right) \prod_{b \neq i, j, k, l}^{n-1} \delta\left(f_{b}^{n-1}\right) .
\end{aligned}
$$

We also need to expand $E_{n}$ in (2.2) to second order in $\lambda$

$$
E_{n}=E_{n}^{(0)}+\lambda E_{n}^{(1)}+\lambda^{2} E_{n}^{(2)}+\mathcal{O}\left(\lambda^{3}\right) .
$$

Plugging (2.7) and (2.10) into (2.2) we get

$$
M_{n}=\frac{1}{\lambda} M_{n}^{(0)}+M_{n}^{(1)}+\lambda M_{n}^{(2)}+\mathcal{O}\left(\lambda^{2}\right),
$$

where

$$
\begin{aligned}
M_{n}^{(0)} & =\int[d \sigma]_{n-4} d \sigma_{n} \delta\left(f_{n}^{n-1}\right) \delta^{(0)} E_{n}^{(0)}, \\
M_{n}^{(1)} & =\int[d \sigma]_{n-4} d \sigma_{n} \delta\left(f_{n}^{n-1}\right)\left(\delta^{(1)} E_{n}^{(0)}+\delta^{(0)} E_{n}^{(1)}\right), \\
M_{n}^{(2)} & =\int[d \sigma]_{n-4} d \sigma_{n} \delta\left(f_{n}^{n-1}\right)\left(\delta^{(2)} E_{n}^{(0)}+\delta^{(1)} E_{n}^{(1)}+\delta^{(0)} E_{n}^{(2)}\right) .
\end{aligned}
$$

The soft theorem conjectures that the following equality should hold

$$
M_{n}^{(i)}=S^{(i)} M_{n-1}, \quad i=0,1,2 .
$$

Weinberg's soft theorem, i.e., $M_{n}^{(0)}=S^{(0)} M_{n-1}$, can be derived as follows. To evaluate $M_{n}^{(0)}$ in $(2.12)$ we also need $E_{n}^{(0)}$, the leading contribution to the determinant (2.4), which is $E_{n}^{(0)}=C_{n n}^{2} E_{n-1}$. In order to see that, we can set $\lambda=0$ in $E_{n}$. Then all the elements of the $(n-2)^{\text {th }}$ row vanish apart from the last one which equals $-C_{n n}$. Similarly all elements of the $(n-2)^{\text {th }}$ column are zero apart from the last one which is $C_{n n}$. Expansion of the determinant along the aforementioned row and column will yield another extra sign which completes the proof. 
Separating all the dependence on $\sigma_{n}$ in $M_{n}^{(0)}$, i.e.,

$$
M_{n}^{(0)}=\int[d \sigma]_{n-4} \delta^{(0)} E_{n-1} \int d \sigma_{n} \delta\left(f_{n}^{n-1}\right) C_{n n}^{2},
$$

we can explicitly evaluate the integral over $\sigma_{n}$. Due to the absence of branch-cuts and the regularity of the integrand when $\sigma_{n} \rightarrow \infty$, we may treat the delta function as a pole and we can evaluate the integral by deforming the contour and using the residue theorem. Performing this one obtains

$$
\int d \sigma_{n} \delta\left(f_{n}^{n-1}\right) C_{n n}^{2}=\sum_{a=1}^{n-1} \frac{\left(\epsilon_{n} \cdot k_{a}\right)^{2}}{k_{n} \cdot k_{a}} .
$$

Putting everything together into (2.14) yields

$$
\begin{aligned}
M_{n}^{(0)} & =\sum_{a=1}^{n-1} \frac{\left(\epsilon_{n} \cdot k_{a}\right)^{2}}{k_{n} \cdot k_{a}} \int[d \sigma]_{n-4} \prod_{l \neq i, j, k}^{n-1} \delta\left(f_{l}^{n-1}\right) E_{n-1} \\
& =\sum_{a=1}^{n-1} \frac{\left(\epsilon_{n} \cdot k_{a}\right)^{2}}{k_{n} \cdot k_{a}} M_{n-1} .
\end{aligned}
$$

From (1.2) one can easily see that $S^{(0)} M_{n-1}$ is precisely the last line of (2.16), thus proving Weinberg's leading soft-graviton theorem.

The computation of (2.13) for $i=1$ in arbitrary dimensions was performed in [21, 22]. In the next section we start the computation of the next to subleading soft contribution $(i=2)$ by evaluating $M_{n}^{(2)}$ in (2.12). Then, in section 4, we will evaluate the action of $S^{(2)}$ on $M_{n-1}$. We will compare both sides of (2.13) by matching terms that contain the same support from the $\delta$-distributions and we will find perfect matching, thus, proving the theorem.

\section{Evaluation of $M_{n}^{(2)}$}

We split the evaluation of $M_{n}^{(2)}$ into three parts

$$
M_{n}^{(2)}=\int[d \sigma]_{n-4}\left(m_{1}+m_{2}+m_{3}\right), \quad m_{i}=\int d \sigma_{n} \delta\left(f_{n}^{n-1}\right) \delta^{(3-i)} E_{n}^{(i-1)} .
$$

\subsection{Evaluation of $m_{1}$}

Using (2.9), the first contribution, $m_{1}$, to $M_{n}^{(2)}$ is

$$
\begin{aligned}
m_{1}= & \frac{1}{2} E_{n-1} \sum_{l \neq i, j, k}^{n-1} \delta^{\prime}\left(f_{l}^{n-1}\right) \sum_{m \neq i, j, k, l}^{n-1} \delta^{\prime}\left(f_{m}^{n-1}\right) \prod_{b \neq i, j, k, l, m}^{n-1} \delta\left(f_{b}^{n-1}\right) I_{1} \\
& +\frac{1}{2} E_{n-1} \sum_{l \neq i, j, k}^{n-1} \delta^{\prime \prime}\left(f_{l}^{n-1}\right) \prod_{b \neq i, j, k, l}^{n-1} \delta\left(f_{b}^{n-1}\right) I_{2},
\end{aligned}
$$


where we have isolated the integration over $\sigma_{n}$ to the following integral

$$
I=k_{l} \cdot k_{n} k_{m} \cdot k_{n} \int d \sigma_{n} \delta\left(f_{n}^{n-1}\right) \frac{C_{n n}^{2}}{\sigma_{n l} \sigma_{n m}} .
$$

Therefore, in (3.2), we have $I_{1}=\left.I\right|_{m \neq l}$ and $I_{2}=\left.I\right|_{m=l}$.

We now move on to compute the integral (3.3). We find

$$
\begin{aligned}
I=\left\{\left[\frac{k_{m} \cdot k_{n} \epsilon_{n} \cdot k_{l}}{\sigma_{m l}}\left(\frac{\epsilon_{n} \cdot k_{l}}{k_{n} \cdot k_{l}} \sum_{c \neq l}^{n-1} \frac{k_{n} \cdot k_{c}}{\sigma_{l c}}-2 \sum_{c \neq l}^{n-1} \frac{\epsilon_{n} \cdot k_{c}}{\sigma_{l c}}\right)-\frac{\left(\epsilon_{n} \cdot k_{l}\right)^{2} k_{m} \cdot k_{n}}{\sigma_{m l}^{2}}\right]+(l \leftrightarrow m)\right. \\
\left.+k_{l} \cdot k_{n} k_{m} \cdot k_{n} \sum_{c \neq l, m}^{n-1} \frac{\left(\epsilon_{n} \cdot k_{c}\right)^{2}}{\sigma_{l c} \sigma_{m c} k_{n} \cdot k_{c}}\right\} \delta_{m \neq l} \\
+\left\{\left(k_{l} \cdot k_{n}\right)^{2} \sum_{c \neq l}^{n-1} \frac{\left(\epsilon_{n} \cdot k_{c}\right)^{2}}{\sigma_{l c}^{2} k_{n} \cdot k_{c}}+\left(\epsilon_{n} \cdot k_{l}\right) \sum_{c \neq l}^{n-1} \frac{\epsilon_{n} \cdot k_{l} k_{n} \cdot k_{c}-2 \epsilon_{n} \cdot k_{c} k_{n} \cdot k_{l}}{\sigma_{l c}^{2}}\right. \\
\left.+k_{l} \cdot k_{n}\left(\sum_{c \neq l}^{n-1} \frac{\epsilon_{n} \cdot k_{c}}{\sigma_{l c}}-\frac{\epsilon_{n} \cdot k_{l}}{k_{n} \cdot k_{l}} \sum_{c \neq l}^{n-1} \frac{k_{n} \cdot k_{c}}{\sigma_{l c}}\right)^{2}\right\} \delta_{m l} .
\end{aligned}
$$

The first line in (3.4) is the contribution of a double pole at $\sigma_{n}=\sigma_{l}$ and a double pole at $\sigma_{n}=\sigma_{m}$, whereas the second line in (3.4) comes from the contribution of a single pole of the integrand at $\sigma_{n}=\sigma_{c}$, for all $c \neq l, m, n$. The first term in the third line comes from a single pole at $\sigma_{n}=\sigma_{c}$ for all $c \neq l, n$ and the remaining of (3.4) comes from a third order pole at $\sigma_{n}=\sigma_{l}$.

\subsection{Evaluation of $m_{2}$}

For the evaluation of $m_{2}$ we need to expand (2.4) to order $\lambda$. The derivative of the determinant of a $n \times n$ matrix with entries $T_{a b}$ can be obtained from the formula

$$
\frac{d}{d \lambda} \operatorname{det}(T)=\sum_{a=1}^{n} \sum_{b=1}^{n}(-1)^{a+b} \frac{d T_{a b}}{d \lambda} M_{b}^{a}
$$

where $M_{b}^{a}$ denotes the determinant of the matrix obtained by removing the $a^{\text {th }}$ row and the $b^{\text {th }}$ column of $T$. Applying it onto $E_{n}$ in equation (2.4) yields

$$
\frac{d E_{n}}{d \lambda}=\sum_{a=1}^{n} \sum_{b=1}^{n}\left((-1)^{a+b} \frac{d A_{a b}}{d \lambda} \tilde{\psi}_{b}^{a}+2(-1)^{a+b+n} \frac{d C_{a b}}{d \lambda} \tilde{\psi}_{b}^{n+a}+(-1)^{a+b} \frac{d B_{a b}}{d \lambda} \tilde{\psi}_{n+b}^{n+a}\right) .
$$

Here we have used the short notation

$$
\tilde{\psi}_{b}^{a} \equiv \frac{4 \operatorname{det}\left(\Psi_{12 b}^{12 a}\right)}{\sigma_{12}^{2}} \delta_{a \neq\{1,2\}} \delta_{b \neq\{1,2\}} .
$$

For convenience and without loss of generality we have chosen to remove the first two rows and the first two columns in (2.4). In (3.6) we have also used the identity $\tilde{\psi}_{b}^{a}=-\tilde{\psi}_{a}^{b}$. The 
derivatives of the different matrix elements are

$$
\begin{aligned}
\frac{d A_{a b}}{d \lambda} & =\frac{1}{\sigma_{a b}}\left(\delta_{a n} k_{n} \cdot k_{b}+\delta_{b n} k_{a} \cdot k_{n}\right) \delta_{a \neq b}, \quad \frac{d B_{a b}}{d \lambda}=0, \\
\frac{d C_{a b}}{d \lambda} & =\frac{\epsilon_{a} \cdot k_{n}}{\sigma_{a b}} \delta_{b n} \delta_{a \neq b}-\delta_{a b} \frac{\epsilon_{a} \cdot k_{n}}{\sigma_{a n}} \delta_{a \neq n} .
\end{aligned}
$$

Putting this into (3.6) yields

$$
\frac{d E_{n}}{d \lambda}=2 \sum_{a=1}^{n-1} \frac{1}{\sigma_{n a}}\left((-1)^{a+n} k_{a} \cdot k_{n} \tilde{\psi}_{a}^{n}+(-1)^{a} \epsilon_{a} \cdot k_{n} \tilde{\psi}_{n+a}^{n}+(-1)^{n-1} \epsilon_{a} \cdot k_{n} \tilde{\psi}_{n+a}^{a}\right) .
$$

Note that all the dependence in $\lambda$ is now contained in the $\tilde{\psi}$ determinants only, which also need to be evaluated at $\lambda=0$ at the end. We further need to isolate any encounter of $\sigma_{n}$ in (3.9), since we eventually want to integrate over that variable. We find

$$
\begin{aligned}
\tilde{\psi}_{a}^{n} & =C_{n n} \sum_{b=1}^{n-1}\left((-1)^{n+b} \frac{\epsilon_{n} \cdot k_{b}}{\sigma_{n b}} \psi_{b}^{a}-(-1)^{b} \frac{\epsilon_{n} \cdot \epsilon_{b}}{\sigma_{n b}} \psi_{n+b-1}^{a}\right), \\
\tilde{\psi}_{n+a}^{n} & =-C_{n n} \sum_{b=1}^{n-1}\left((-1)^{n+b} \frac{\epsilon_{n} \cdot k_{b}}{\sigma_{n b}} \psi_{b}^{n+a-1}-(-1)^{b} \frac{\epsilon_{n} \cdot \epsilon_{b}}{\sigma_{n b}} \psi_{n+b-1}^{n+a-1}\right), \\
\tilde{\psi}_{n+a}^{a} & =-C_{n n}^{2} \psi_{n+a-1}^{a},
\end{aligned}
$$

where we have dropped the tilde sign to denote the further removal of the rows and columns that contain the variable $\sigma_{n}$, that is $\psi_{b}^{a}$ denotes the determinant $E_{n-1}$ after the removal of the $a^{\text {th }}$ row and the $b^{\text {th }}$ column. Then

$$
\begin{aligned}
E_{n}^{(1)}= & 2 C_{n n} \sum_{a=1}^{n-1} \sum_{b=1}^{n-1} \frac{(-1)^{a+b}}{\sigma_{n a} \sigma_{n b}}\left(k_{n} \cdot k_{a} \epsilon_{n} \cdot k_{b} \psi_{b}^{a}-k_{n} \cdot \epsilon_{b} \epsilon_{n} \cdot \epsilon_{a} \psi_{n+b-1}^{n+a-1}\right. \\
& \left.+(-1)^{n}\left(\epsilon_{n} \cdot k_{a} k_{n} \cdot \epsilon_{b}-k_{n} \cdot k_{a} \epsilon_{n} \cdot \epsilon_{b}\right) \psi_{n+b-1}^{a}\right) \\
& +2 C_{n n}^{2} \sum_{a=1}^{n-1} \frac{(-1)^{n}}{\sigma_{n a}} \epsilon_{a} \cdot k_{n} \psi_{n+a-1}^{a} .
\end{aligned}
$$

We recall that $m_{2}$ takes the form

$$
m_{2}=\int d \sigma_{n} \delta\left(f_{n}^{n-1}\right) \sum_{l \neq i, j, k}^{n-1} \frac{k_{n} \cdot k_{l}}{\sigma_{l n}} \delta^{\prime}\left(f_{l}^{n-1}\right) \prod_{m \neq i, j, k, l}^{n-1} \delta\left(f_{m}^{n-1}\right) E_{n}^{(1)}
$$

thus, we will need the following integrals

$$
\begin{aligned}
I_{3} & \equiv-k_{n} \cdot k_{l} \int d \sigma_{n} \delta\left(f_{n}^{n-1}\right) \frac{C_{n n}}{\sigma_{n l} \sigma_{n a} \sigma_{n b}}, \\
I_{4} & \equiv-k_{n} \cdot k_{l} \int d \sigma_{n} \delta\left(f_{n}^{n-1}\right) \frac{C_{n n}^{2}}{\sigma_{n l} \sigma_{n a}} .
\end{aligned}
$$


The integral $I_{4}$ is directly obtained from (3.4) since $I_{4}=-\left.\left(k_{n} \cdot k_{a}\right)^{-1} I\right|_{m=a}$. For $I_{3}$ we find

$$
\begin{aligned}
I_{3}= & k_{n} \cdot k_{l}\left[\frac{\epsilon_{n} \cdot k_{a}}{k_{n} \cdot k_{a}} \frac{1}{\sigma_{a l} \sigma_{a b}}+(a \leftrightarrow l)+(a \leftrightarrow b)\right] \delta_{l \neq a} \delta_{l \neq b} \delta_{a \neq b} \\
& +k_{n} \cdot k_{l}\left[\left(\frac{\epsilon_{n} \cdot k_{a}}{k_{n} \cdot k_{a}} \frac{1}{\sigma_{a l}} \sum_{c \neq a}^{n-1} \frac{1}{\sigma_{a c}}\left(\frac{\epsilon_{n} \cdot k_{c}}{\epsilon_{n} \cdot k_{a}}-\frac{k_{n} \cdot k_{c}}{k_{n} \cdot k_{a}}\right)+\frac{\epsilon_{n} \cdot k_{l}}{k_{n} \cdot k_{l}} \frac{1}{\sigma_{a l}^{2}}-\frac{\epsilon_{n} \cdot k_{a}}{k_{n} \cdot k_{a}} \frac{1}{\sigma_{a l}^{2}}\right) \delta_{l \neq a} \delta_{a b}\right. \\
& +(l \leftrightarrow a)+(\{a, l, b\} \rightarrow\{l, b, a\})] \\
+ & \epsilon_{n} \cdot k_{l}\left[-\frac{1}{\epsilon_{n} \cdot k_{l}} \sum_{c \neq l}^{n-1} \frac{\epsilon_{n} \cdot k_{c}}{\sigma_{l c}^{2}}-\frac{1}{k_{n} \cdot k_{l} \epsilon_{n} \cdot k_{l}} \sum_{c \neq l}^{n-1} \sum_{d \neq l}^{n-1} \frac{k_{n} \cdot k_{c} \epsilon_{n} \cdot k_{d}}{\sigma_{l c} \sigma_{l d}}\right. \\
& \left.+\frac{1}{k_{n} \cdot k_{l}} \sum_{c \neq l}^{n-1} \frac{k_{n} \cdot k_{c}}{\sigma_{l c}^{2}}+\frac{1}{\left(k_{n} \cdot k_{l}\right)^{2}}\left(\sum_{c \neq l}^{n-1} \frac{k_{n} \cdot k_{c}}{\sigma_{l c}}\right)^{2}\right] \delta_{a b} \delta_{b l} .
\end{aligned}
$$

As a check, note that from this expression the quantity $I_{3} /\left(k_{n} \cdot k_{l}\right)$ is symmetric under the exchange of any two pairs of $(l, a, b)$ which is evident from the original definition in (3.13).

We now write $m_{2}$ making explicit the linear combination of the different types of minors we have, i.e.,

$$
m_{2}=2 \sum_{l \neq i, j, k}^{n-1} \delta^{\prime}\left(f_{l}^{n-1}\right) \prod_{m \neq i, j, k, l}^{n-1} \delta\left(f_{m}^{n-1}\right) D_{l}
$$

where

$$
\begin{aligned}
D_{l} \equiv & \sum_{a \neq l}^{n-1} \sum_{b \neq l, a}^{n-1}\left(c_{1} \psi_{b}^{a}+c_{2} \psi_{n+b-1}^{n+a-1}+c_{3} \psi_{n+b-1}^{a}\right) I_{3\{l \neq a, l \neq b, a \neq b\}} \\
& +\sum_{a \neq l}^{n-1} c_{4} \psi_{n+a-1}^{a}+\sum_{a \neq l}^{n-1}\left(c_{5} \psi_{l}^{a}+c_{6} \psi_{n+l-1}^{n+a-1}+c_{7} \psi_{n+l-1}^{a}+c_{8} \psi_{n+a-1}^{l}\right) I_{3\{l=b, l \neq a\}} \\
& +c_{9} \psi_{l+n-1}^{l} .
\end{aligned}
$$

The coefficients $c_{i}$ are

$$
\begin{aligned}
& c_{1}=(-1)^{a+b} k_{n} \cdot k_{a} \epsilon_{n} \cdot k_{b} ; c_{2}=-(-1)^{a+b} \epsilon_{b} \cdot k_{n} \epsilon_{n} \cdot \epsilon_{a} ; c_{3}=(-1)^{n}\left(\epsilon_{n} \cdot k_{a} \epsilon_{b} \cdot k_{n}-k_{n} \cdot k_{a} \epsilon_{n} \cdot \epsilon_{b}\right) ; \\
& c_{4}=(-1)^{n}\left(\epsilon_{n} \cdot k_{a} k_{n} \cdot \epsilon_{a}-k_{n} \cdot k_{a} \epsilon_{n} \cdot \epsilon_{a}\right) I_{3\{a=b, l \neq a\}}+(-1)^{n} \epsilon_{a} \cdot k_{n} I_{4\{l \neq a\}} ; \\
& c_{5}=(-1)^{a+l}\left(k_{n} \cdot k_{a} \epsilon_{n} \cdot k_{l}-k_{n} \cdot k_{l} \epsilon_{n} \cdot k_{a}\right) ; c_{6}=(-1)^{a+l}\left(\epsilon_{a} \cdot k_{n} \epsilon_{n} \cdot \epsilon_{l}-\epsilon_{l} \cdot k_{n} \epsilon_{n} \cdot \epsilon_{a}\right) ; \\
& c_{7}=(-1)^{a+l+n}\left(\epsilon_{n} \cdot k_{a} \epsilon_{l} \cdot k_{n}-k_{n} \cdot k_{a} \epsilon_{n} \cdot \epsilon_{l}\right) ; c_{8}=(-1)^{a+l+n}\left(\epsilon_{n} \cdot k_{l} \epsilon_{a} \cdot k_{n}-k_{n} \cdot k_{l} \epsilon_{n} \cdot \epsilon_{a}\right) ; \\
& c_{9}=(-1)^{n}\left(\epsilon_{n} \cdot k_{l} k_{n} \cdot \epsilon_{l}-k_{n} \cdot k_{l} \epsilon_{n} \cdot \epsilon_{l}\right) I_{3\{l=a=b\}}+(-1)^{n} \epsilon_{l} \cdot k_{n} I_{4\{l=a\}} .
\end{aligned}
$$

In the above we have used the identity $\psi_{b}^{a}=-\psi_{a}^{b}$.

\subsection{Evaluation of $m_{3}$}

We define $\tilde{\psi}_{c d}^{a b}$ and $\psi_{c d}^{a b}$ to be respectively the determinants $E_{n}$ and $E_{n-1}$ after the removal of the rows $a, b$ and the columns $c, d$. 
For the evaluation of $m_{3}$ we need to take the second derivative of (2.4) with respect to $\lambda$. From (3.9) we have

$$
\frac{d^{2} E_{n}}{d \lambda^{2}}=2 \sum_{a=1}^{n-1} \frac{1}{\sigma_{n a}}\left((-1)^{a+n} k_{a} \cdot k_{n} \frac{d \tilde{\psi}_{a}^{n}}{d \lambda}+(-1)^{a} \epsilon_{a} \cdot k_{n} \frac{d \tilde{\psi}_{n+a}^{n}}{d \lambda}+(-1)^{n-1} \epsilon_{a} \cdot k_{n} \frac{d \tilde{\psi}_{n+a}^{a}}{d \lambda}\right) .
$$

With the definition $\theta_{i j}$ to be 0 when $i>j$ and -1 when $i<j$ we find

$$
\begin{aligned}
\frac{d \tilde{\psi}_{a}^{n}}{d \lambda}= & \sum_{b=1}^{n-1} \frac{1}{\sigma_{b n}}\left((-1)^{n+b-1} k_{b} \cdot k_{n} \tilde{\psi}_{a n}^{b n}+(-1)^{n-1} \epsilon_{b} \cdot k_{n} \tilde{\psi}_{a, n+b}^{b n}+(-1)^{b} \epsilon_{b} \cdot k_{n} \tilde{\psi}_{a n}^{n, n+b}\right) \\
& +\sum_{b \neq a}^{n-1}(-1)^{n+\theta_{a b}} \frac{\epsilon_{b} \cdot k_{n}}{\sigma_{b n}} \tilde{\psi}_{a b}^{n, n+b}, \\
\frac{d \tilde{\psi}_{n+a}^{n}}{d \lambda}= & \sum_{b=1}^{n-1} \frac{1}{\sigma_{b n}}\left((-1)^{n+b} k_{b} \cdot k_{n} \tilde{\psi}_{n, n+a}^{b n}+(-1)^{b-1} \epsilon_{b} \cdot k_{n} \tilde{\psi}_{n, n+a}^{n, n+b}+(-1)^{n} \epsilon_{b} \cdot k_{n} \tilde{\psi}_{b, n+a}^{n, n+b}\right) \\
& +\sum_{b \neq a}^{n-1}(-1)^{n+\theta_{a b}} \frac{\epsilon_{b} \cdot k_{n}}{\sigma_{b n}} \tilde{\psi}_{n+a, n+b}^{b n}, \\
\frac{d \tilde{\psi}_{n+a}^{a}=}{d \lambda} & \sum_{b=1}^{n-1} \frac{1}{\sigma_{b n}}\left((-1)^{n+b} k_{b} \cdot k_{n} \tilde{\psi}_{b, n+a}^{a n}+(-1)^{n} \epsilon_{b} \cdot k_{n} \tilde{\psi}_{b, n+a}^{a, n+b}+(-1)^{b-1} \epsilon_{b} \cdot k_{n} \tilde{\psi}_{n, n+a}^{a, n+b}\right) \\
& +\sum_{b \neq a}^{n-1} \frac{(-1)^{\theta}}{\sigma_{b n}}\left((-1)^{n+b} k_{b} \cdot k_{n} \tilde{\psi}_{n, n+a}^{a b}\right. \\
& \left.+(-1)^{n+\theta_{a b}} \epsilon_{b} \cdot k_{n} \tilde{\psi}_{n+a, n+b}^{a b}+(-1)^{b} \epsilon_{b} \cdot k_{n} \tilde{\psi}_{n+a, n+b}^{a n}\right) .
\end{aligned}
$$

We can further expand the $n$ and $2 n$ rows and columns of the minors appearing in (3.20). With the help of the identity $\psi_{c d}^{a b}=\psi_{a b}^{c d}$ we arrive at the following result

$$
E_{n}^{(2)}=C_{n n}^{2} A_{1}+C_{n n} A_{2}+A_{3},
$$

where

$$
\begin{aligned}
A_{1}= & \sum_{a=1}^{n-1} \sum_{b=1}^{n-1} \frac{\epsilon_{a} \cdot k_{n} \epsilon_{b} \cdot k_{n}}{\sigma_{n a} \sigma_{n b}} \psi_{b, n+a-1}^{a, n+b-1}+\sum_{a=1}^{n-1} \sum_{b \neq a}^{n-1} \frac{\epsilon_{a} \cdot k_{n} \epsilon_{b} \cdot k_{n}}{\sigma_{n a} \sigma_{n b}} \psi_{n+a-1, n+b-1}^{a b} \\
A_{2}= & 2 \sum_{a=1}^{n-1} \sum_{b=1}^{n-1} \sum_{c=1}^{n-1} \frac{(-1)^{b+c}}{\sigma_{n a} \sigma_{n b} \sigma_{n c}} \epsilon_{a} \cdot k_{n}\left(\epsilon_{b} \cdot k_{n} \epsilon_{n} \cdot k_{c}-k_{c} \cdot k_{n} \epsilon_{b} \epsilon_{n}\right) \psi_{c, n+a-1}^{a, n+b-1} \\
& +2 \sum_{a=1}^{n-1} \sum_{c=1}^{n-1} \sum_{b \neq a}^{n-1} \frac{(-1)^{b+c+n+\theta_{a b}}}{\sigma_{n a} \sigma_{n b} \sigma_{n c}} \epsilon_{a} \cdot k_{n} \\
& {\left[\left(k_{b} \cdot k_{n} \epsilon_{n} \cdot k_{c}-k_{c} k_{n} \epsilon_{n} \cdot k_{b}\right) \psi_{c, n+a-1}^{a b}+\left(\epsilon_{b} \cdot k_{n} \epsilon_{c} \cdot \epsilon_{n}-\epsilon_{c} \cdot k_{n} \epsilon_{b} \cdot \epsilon_{n}\right) \psi_{n+a-1, n+b-1}^{a, n+c-1}\right] } \\
& +2 \sum_{a=1}^{n-1} \sum_{b \neq a}^{n-1} \sum_{c \neq a}^{n-1} \frac{(-1)^{b+c+\theta_{a b}+\theta_{a c}}}{\sigma_{n a} \sigma_{n b} \sigma_{n c}} \epsilon_{a} \cdot k_{n}\left(\epsilon_{c} \cdot k_{n} \epsilon_{n} \cdot k_{b}-k_{b} \cdot k_{n} \epsilon_{c} \cdot \epsilon_{n}\right) \psi_{n+a-1, n+c-1}^{a b}
\end{aligned}
$$




$$
\begin{aligned}
& A_{3}= \sum_{a=1}^{n-1} \sum_{b=1}^{n-1} \sum_{c=1}^{n-1} \sum_{d=1}^{n-1} \frac{(-1)^{a+b+c+d}}{\sigma_{n a} \sigma_{n b} \sigma_{n c} \sigma_{n d}}\left(\epsilon_{n} \cdot k_{a} \epsilon_{n} \cdot k_{b} \epsilon_{c} \cdot k_{n} \epsilon_{d} \cdot k_{n}+k_{a} \cdot k_{n} k_{b} \cdot k_{n} \epsilon_{c} \cdot \epsilon_{n} \epsilon_{d} \cdot \epsilon_{n}\right. \\
&\left.-2 k_{a} \cdot k_{n} \epsilon_{n} \cdot k_{b} \epsilon_{c} \cdot k_{n} \epsilon_{d} \cdot \epsilon_{n}\right) \psi_{a, n+d-1}^{b, n+c-1}
\end{aligned}
$$

In order to finish the calculation of $m_{3}$ the only new integral we need to evaluate is

$$
I_{5} \equiv \int d \sigma_{n} \delta\left(f_{n}^{n-1}\right) \frac{1}{\sigma_{n a} \sigma_{n b} \sigma_{n c} \sigma_{n d}}
$$

for which we obtain

$$
\begin{aligned}
I_{5}= & \frac{1}{\left(k_{n} \cdot k_{a}\right)^{2}}\left[\sum_{l \neq a}^{n-1} \frac{k_{n} \cdot k_{l}}{\sigma_{a l}^{2}}+\frac{1}{k_{n} \cdot k_{a}}\left(\sum_{l \neq a}^{n-1} \frac{k_{n} \cdot k_{l}}{\sigma_{a l}}\right)^{2}\right] \delta_{a b} \delta_{b c} \delta_{c d} \\
& +\left\{\frac{1}{k_{n} \cdot k_{b}} \frac{1}{\sigma_{a b}}\left[\frac{1}{k_{n} \cdot k_{b}} \sum_{l \neq b}^{n-1} \frac{k_{n} \cdot k_{l}}{\sigma_{b l}}-\frac{1}{\sigma_{a b}}\right] \delta_{a \neq b} \delta_{b c} \delta_{c d}+\operatorname{cyclic}\{a, b, c, d\}\right\} \\
& +\left\{\left(\frac{1}{k_{n} \cdot k_{a}}+\frac{1}{k_{n} \cdot k_{c}}\right) \frac{1}{\sigma_{a c}^{2}} \delta_{a b} \delta_{c d} \delta_{a \neq c}+\operatorname{cyclic}\{b, c, d\}\right\} \\
& +\left\{\frac{1}{\sigma_{a c} \sigma_{a d}} \frac{1}{k_{n} \cdot k_{a}} \delta_{a b} \delta_{c \neq d} \delta_{a \neq c, d}+\operatorname{cyclic}\{b, c, d\}\right\} \\
& +\left\{\frac{1}{\sigma_{b a} \sigma_{b d}} \frac{1}{k_{n} \cdot k_{b}} \delta_{b c} \delta_{a \neq d} \delta_{b \neq a, d}+\operatorname{cyclic}\{b, c, d\}\right\}
\end{aligned}
$$

\section{Action of $S^{(2)}$ on the amplitude}

From (1.3), the complete expression for $S^{(2)}$ including the spin contribution can be written as

$$
S^{(2)}=S_{\mathrm{orb}}^{(2)}+S_{\mathrm{so}}^{(2)}+S_{\mathrm{spin}}^{(2)}
$$

where the orbital, spin-orbit and spin parts are respectively given by

$$
S_{\mathrm{orb}}^{(2)}=\frac{1}{2} \sum_{a=1}^{n-1} K_{a \mu \nu}^{\mathrm{orb}} \frac{\partial^{2}}{\partial k_{a \mu} \partial k_{a \nu}}, \quad S_{\mathrm{so}}^{(2)}=\sum_{a=1}^{n-1} K_{a \mu \nu}^{\mathrm{so}} \frac{\partial^{2}}{\partial k_{a \mu} \partial \epsilon_{a \nu}}, \quad S_{\mathrm{spin}}^{(2)}=\frac{1}{2} \sum_{a=1}^{n-1} K_{a \mu \nu}^{\mathrm{spin}} \frac{\partial^{2}}{\partial \epsilon_{a \mu} \partial \epsilon_{a \nu}},
$$


with

$$
\begin{aligned}
& K_{a \mu \nu}^{\mathrm{orb}} \equiv k_{n} \cdot k_{a} \epsilon_{n \mu} \epsilon_{n \nu}-\epsilon_{n} \cdot k_{a} \epsilon_{n(\mu} k_{n \nu}+\frac{\left(\epsilon_{n} \cdot k_{a}\right)^{2}}{k_{n} \cdot k_{a}} k_{n \mu} k_{n \nu} \\
& K_{a \mu \nu}^{\mathrm{so}} \equiv \epsilon_{a} \cdot k_{n} \epsilon_{n \mu} \epsilon_{n \nu}-\epsilon_{n} \cdot \epsilon_{a} \epsilon_{n \mu} k_{n \nu}-\frac{\epsilon_{n} \cdot k_{a} \epsilon_{a} \cdot k_{n}}{k_{n} \cdot k_{a}} \epsilon_{n \nu} k_{n \mu}+\frac{\epsilon_{n} \cdot k_{a} \epsilon_{n} \cdot \epsilon_{a}}{k_{n} \cdot k_{a}} k_{n \mu} k_{n \nu}, \\
& \left.K_{a \mu \nu}^{\mathrm{spin}} \equiv \frac{\left(\epsilon_{a} \cdot k_{n}\right)^{2}}{k_{n} \cdot k_{a}} \epsilon_{n \mu} \epsilon_{n \nu}-\frac{\epsilon_{a} \cdot k_{n} \epsilon_{n} \cdot \epsilon_{a}}{k_{n} \cdot k_{a}} \epsilon_{n(\mu} k_{n \nu}\right)+\frac{\left(\epsilon_{n} \cdot \epsilon_{a}\right)^{2}}{k_{n} \cdot k_{a}} k_{n \mu} k_{n \nu} .
\end{aligned}
$$

Then the action of $S^{(2)}$ on the amplitude is

$$
\begin{aligned}
S^{(2)} M_{n-1} & =S^{(2)} \int[d \sigma]_{n-4} \prod_{l \neq i, j, k}^{n-1} \delta\left(f_{l}^{n-1}\right) E_{n-1} \\
& =\int[d \sigma]_{n-4}\left(s_{1}+s_{2}+s_{3}+s_{4}\right),
\end{aligned}
$$

where we have separated the calculation into the following four parts

$$
\begin{array}{rlrl}
s_{1} & =E_{n-1} S_{\mathrm{orb}}^{(2)} \prod_{l \neq i, j, k}^{n-1} \delta\left(f_{l}^{n-1}\right), & s_{2} & =\sum_{a=1}^{n-1} K_{a \mu \nu}^{\mathrm{orb}} \frac{\partial E_{n-1}}{\partial k_{a \mu}} \frac{\partial}{\partial k_{a \nu}} \prod_{l \neq i, j, k}^{n-1} \delta\left(f_{l}^{n-1}\right), \\
s_{3}=\sum_{a=1}^{n-1} K_{a \mu \nu}^{\mathrm{so}} \frac{\partial E_{n-1}}{\partial \epsilon_{a \nu}} \frac{\partial}{\partial k_{a \mu}} \prod_{l \neq i, j, k}^{n-1} \delta\left(f_{l}^{n-1}\right), & s_{4}=\prod_{l \neq i, j, k}^{n-1} \delta\left(f_{l}^{n-1}\right) S^{(2)} E_{n-1} .
\end{array}
$$

In the subsequent computations we will make use of the identities

$$
\frac{\partial f_{l}^{n}}{\partial k_{a \mu}}=\frac{k_{l}^{\mu}}{\sigma_{l a}} \delta_{l \neq a}+\delta_{l a} \sum_{d \neq l}^{n} \frac{k_{d}^{\mu}}{\sigma_{l d}}, \quad \frac{\partial^{2} f_{l}^{n}}{\partial k_{a \mu} \partial k_{a \nu}}=0,
$$

and also

$$
\begin{aligned}
& \frac{\partial E_{n-1}}{\partial k_{a \mu}}=2 \sum_{b \neq a}^{n-1} \frac{1}{\sigma_{a b}}\left((-1)^{a+b} k_{b}{ }^{\mu} \psi_{b}^{a}+(-1)^{a+b+n+1} \epsilon_{b}{ }^{\mu} \psi_{n+b-1}^{a}+(-1)^{n} \epsilon_{b}{ }^{\mu} \psi_{n+b-1}^{b}\right), \\
& \frac{\partial E_{n-1}}{\partial \epsilon_{a \mu}}=2 \sum_{b \neq a}^{n-1} \frac{1}{\sigma_{a b}}\left((-1)^{a+b+n} k_{b}{ }^{\mu} \psi_{n+a-1}^{b}+(-1)^{n+1} k_{b}{ }^{\mu} \psi_{n+a-1}^{a}+(-1)^{a+b} \epsilon_{b}{ }^{\mu} \psi_{n+b-1}^{n+a-1}\right) .
\end{aligned}
$$

In the following we omit the upper index of the scattering equations $f_{l}^{n-1}$ and we simply write them as $f_{l}$.

\subsection{Evaluation of $s_{1}$}

We find

$$
\begin{aligned}
s_{1}= & \frac{1}{2} E_{n-1} \sum_{l \neq i, j, k}^{n-1} \delta^{\prime}\left(f_{l}\right) \sum_{m \neq i, j, k, l}^{n-1} \delta^{\prime}\left(f_{m}\right) \prod_{b \neq i, j, k, l, m}^{n-1} \delta\left(f_{b}\right) \sum_{a=1}^{n-1} K_{a \mu \nu}^{\mathrm{orb}} \frac{\partial f_{m}}{\partial k_{a \mu}} \frac{\partial f_{l}}{\partial k_{a \nu}} \\
& +\frac{1}{2} E_{n-1} \sum_{l \neq i, j, k}^{n-1} \delta^{\prime \prime}\left(f_{l}\right) \prod_{b \neq i, j, k, l}^{n-1} \delta\left(f_{b}\right) \sum_{a=1}^{n-1} K_{a \mu \nu}^{\mathrm{orb}} \frac{\partial f_{l}}{\partial k_{a \mu}} \frac{\partial f_{l}}{\partial k_{a \nu}} .
\end{aligned}
$$


After some straightforward algebra and using (4.3) and (4.6) we obtain

$$
\sum_{a=1}^{n-1} K_{a \mu \nu}^{\mathrm{orb}} \frac{\partial f_{m}}{\partial k_{a \mu}} \frac{\partial f_{l}}{\partial k_{a \nu}}=\delta_{m l} I_{2}+\delta_{m \neq l} I_{1}
$$

thus, comparing with (3.2), we obtain the desired result $s_{1}=m_{1}$.

\subsection{Evaluation of $s_{2}$ and $s_{3}$}

The combination $s_{2}+s_{3}$ has the same delta function support as $m_{2}$, thus, we will compare these two expressions. For $s_{2}$ we obtain

$$
s_{2}=\sum_{l \neq i, j, k}^{n-1} \delta^{\prime}\left(f_{l}\right) \prod_{m \neq i, k, j, l}^{n-1} \delta\left(f_{m}\right) \sum_{a=1}^{n-1} K_{a \mu \nu}^{\mathrm{orb}} \frac{\partial E_{n-1}}{\partial k_{a \mu}} \frac{\partial f_{l}}{\partial k_{a \nu}}
$$

and for $s_{3}$ we get

$$
s_{3}=\sum_{l \neq i, j, k}^{n-1} \delta^{\prime}\left(f_{l}\right) \prod_{m \neq i, k, j, l}^{n-1} \delta\left(f_{m}\right) \sum_{a=1}^{n-1} K_{a \mu \nu}^{\mathrm{so}} \frac{\partial E_{n-1}}{\partial \epsilon_{a \nu}} \frac{\partial f_{l}}{\partial k_{a \mu}} .
$$

After some tedious but straightforward algebra and using (4.3), (4.6) and (4.7) we can expand $s_{2}+s_{3}$ in the same form of $m_{2}$ as shown in (3.16) and (3.17). We have explicitly computed each of the coefficients of the corresponding expansion for $s_{2}+s_{3}$ and see that they all precisely match those of (3.18), thus, arriving at $s_{2}+s_{3}=m_{2}$ as expected.

\subsection{Evaluation of $s_{4}$}

Having matched all the previous terms on both sides, our last task is to show that $s_{4}=m_{3}$.

From (4.5), (4.1) and (4.2) we have

$$
s_{4}=\prod_{l \neq i, j, k}^{n-1} \delta\left(f_{l}^{n-1}\right) \sum_{a=1}^{n-1}\left[\frac{1}{2} K_{a \mu \nu}^{\mathrm{orb}} \frac{\partial^{2} E_{n-1}}{\partial k_{a \nu} \partial k_{a \mu}}+K_{a \mu \nu}^{\mathrm{so}} \frac{\partial^{2} E_{n-1}}{\partial \epsilon_{a \nu} \partial k_{a \mu}}+\frac{1}{2} K_{a \mu \nu}^{\mathrm{spin}} \frac{\partial^{2} E_{n-1}}{\partial \epsilon_{a \nu} \partial \epsilon_{a \mu}}\right] .
$$

Appropriately differentiating (4.7) we find

$$
\begin{aligned}
& \frac{\partial^{2} E_{n-1}}{\partial k_{a \nu} \partial k_{a \mu}}=2 \sum_{b \neq a}^{n-1} \sum_{c \neq a}^{n-1} \frac{1}{\sigma_{a b} \sigma_{c a}}\left((-1)^{b+c+\theta_{b a}+\theta_{a c} k_{b}{ }^{\mu} k_{c}{ }^{\nu} \psi_{a b}^{a c}}\right. \\
& +(-1)^{a+b+n+\theta_{a c}}\left(k_{b}{ }^{\mu} \epsilon_{c}{ }^{\nu}+\epsilon_{c}{ }^{\mu} k_{b}{ }^{\nu}\right) \psi_{b, n+c-1}^{a c}+(-1)^{b+c+n+\theta_{b a}}\left(k_{b}{ }^{\mu} \epsilon_{c}{ }^{\nu}+\epsilon_{c}{ }^{\mu} k_{b}{ }^{\nu}\right) \psi_{a, n+c-1}^{a b} \\
& \left.+(-1)^{a+b}\left(\epsilon_{b}{ }^{\mu} \epsilon_{c}{ }^{\nu}+\epsilon_{c}{ }^{\mu} \epsilon_{b}{ }^{\nu}\right) \psi_{c, n+b-1}^{a, n+c-1}-\epsilon_{b}{ }^{\mu} \epsilon_{c}{ }^{\nu}\left(\psi_{c, n+b-1}^{b, n+c-1}+(-1)^{b+c} \psi_{a, n+b-1}^{a, n+c-1}\right)\right) \\
& \quad+2 \sum_{b \neq a}^{n-1} \sum_{c \neq a, b}^{n-1} \frac{1}{\sigma_{a b} \sigma_{c a}}\left((-1)^{a+b+n+\theta_{c b}}\left(k_{b}{ }^{\mu} \epsilon_{c}{ }^{\nu}+\epsilon_{c}{ }^{\mu} k_{b}{ }^{\nu}\right) \psi_{a, n+c-1}^{b c}\right. \\
& \left.+(-1)^{a+b+\theta_{b c}+\theta_{a c}}\left(\epsilon_{b}{ }^{\mu} \epsilon_{c}{ }^{\nu}+\epsilon_{c}{ }^{\mu} \epsilon_{b}{ }^{\nu}\right) \psi_{n+b-1, n+c-1}^{a c}-\epsilon_{b}{ }^{\mu} \epsilon_{c}{ }^{\nu} \psi_{n+b-1, n+c-1}^{b c}\right), \\
& \frac{\partial^{2} E_{n-1}}{\partial \epsilon_{a \nu} \partial k_{a \mu}}=2 \sum_{b \neq a}^{n-1} \sum_{c \neq a}^{n-1} \frac{1}{\sigma_{a b} \sigma_{a c}}\left[(-1)^{b+n} k_{b}{ }^{\mu} k_{c}{ }^{\nu}\left((-1)^{c+\theta_{c a}} \psi_{b, n+a-1}^{a c}+(-1)^{a+\theta_{a b}} \psi_{a, n+a-1}^{a b}\right)\right.
\end{aligned}
$$




$$
\begin{aligned}
& +(-1)^{b+c}\left(k_{b}{ }^{\mu} \epsilon_{c}{ }^{\nu}-\epsilon_{c}{ }^{\mu} k_{b}{ }^{\nu}\right) \psi_{b, n+c-1}^{a, n+a-1}-(-1)^{b+c} k_{b}{ }^{\mu} \epsilon_{c}{ }^{\nu} \psi_{b, n+a-1}^{a, n+c-1} \\
& +\epsilon_{b}{ }^{\mu} k_{c}{ }^{\nu}\left((-1)^{b+c+\theta_{a b}+\theta_{a c}} \psi_{n+a-1, n+b-1}^{a c}-\psi_{n+a-1, n+b-1}^{a b}\right) \\
& +\epsilon_{b}{ }^{\mu} k_{c}{ }^{\nu}\left((-1)^{a+b} \psi_{a, n+b-1}^{a, n+a-1}+(-1)^{a+c} \psi_{c, n+b-1}^{b, n+a-1}-\psi_{a, n+b-1}^{b, n+a-1}\right) \\
& \left.+\epsilon_{b}{ }^{\mu} \epsilon_{c}{ }^{\nu}(-1)^{c+n+\theta_{a b}}\left((-1)^{b} \psi_{n+a-1, n+b-1}^{a, n+c-1}-(-1)^{a} \psi_{n+a-1, n+b-1}^{b, n+c-1}\right)\right] \\
& +2 \sum_{b \neq a}^{n-1} \sum_{c \neq a, b}^{n-1} \frac{1}{\sigma_{a b} \sigma_{a c}}\left[(-1)^{b+c+n+\theta_{b c}} k_{b}{ }^{\mu} k_{c}{ }^{\nu} \psi_{a, n+a-1}^{b c}\right. \\
& +(-1)^{a+c+n+\theta_{c b}} \epsilon_{b}{ }^{\mu} \epsilon_{c}{ }^{\nu} \psi_{n+b-1, n+c-1}^{b, n+a-1}+(-1)^{b+c+n+\theta_{b c}} \epsilon_{b}{ }^{\mu} \epsilon_{c}{ }^{\nu} \psi_{n+b-1, n+c-1}^{a, n+a-1} \\
& \left.+(-1)^{a+c+\theta_{b a}+\theta_{b c}} \epsilon_{b}{ }^{\mu} k_{c}{ }^{\nu} \psi_{n+a-1, n+b-1}^{b c}\right] \text {, } \\
& \frac{\partial^{2} E_{n-1}}{\partial \epsilon_{a \nu} \partial \epsilon_{a \mu}}=2 \sum_{b \neq a}^{n-1} \sum_{c \neq a}^{n-1} \frac{1}{\sigma_{a b} \sigma_{a c}}\left[k_{b}{ }^{\mu} k_{c}{ }^{\nu}\left((-1)^{b+c} \psi_{c, n+a-1}^{b, n+a-1}+\psi_{a, n+a-1}^{a, n+a-1}\right)\right. \\
& +(-1)^{b+c+\theta_{b a}+\theta_{c a}} \epsilon_{b}{ }^{\mu} \epsilon_{c}{ }^{\nu} \psi_{n+a-1, n+c-1}^{n+a-1, n+b-1}-(-1)^{a+b}\left(k_{b}{ }^{\mu} k_{c}{ }^{\nu}+k_{c}{ }^{\mu} k_{b}{ }^{\nu}\right) \psi_{b, n+a-1}^{a, n+a-1} \\
& \left.+(-1)^{c+n+\theta_{c a}}\left(k_{b}{ }^{\mu} \epsilon_{c}{ }^{\nu}+\epsilon_{c}{ }^{\mu} k_{b}{ }^{\nu}\right)\left((-1)^{b} \psi_{n+a-1, n+c-1}^{b, n+a-1}-(-1)^{a} \psi_{n+a-1, n+c-1}^{a, n+a-1}\right)\right] .
\end{aligned}
$$

We now have all the ingredients to perform the comparison of $s_{4}$ with $m_{3}$. The algebra is tedious but straightforward since it only involves changes of the summation order and renaming dummy indices. We have performed the analysis and found agreement of the two expressions which completes the proof of the soft-graviton theorem.

\section{Acknowledgments}

We thank Wei He for useful discussions. We also thank Anastasia Volovich and Michael Zlotnikov for correspondence. In the final stages of the writing of this paper we learned of the work of Michael Zlotnikov [32] who was also embarked on our same computations. C.K. would like to thank the organizers of the Simons workshop during which this project was completed. The work of C.K. is supported by the São Paulo Research Foundation (FAPESP) under grants 2011/11973-4 and 2012/00756-5. The work of F.R is supported by FAPESP grant 2012/05451-8.

Open Access. This article is distributed under the terms of the Creative Commons Attribution License (CC-BY 4.0), which permits any use, distribution and reproduction in any medium, provided the original author(s) and source are credited.

\section{References}

[1] S. Weinberg, Photons and gravitons in s matrix theory: derivation of charge conservation and equality of gravitational and inertial mass, Phys. Rev. 135 (1964) B1049 [InSPIRE].

[2] S. Weinberg, Infrared photons and gravitons, Phys. Rev. 140 (1965) B516 [InSPIRE]. 
[3] A. Strominger, On BMS invariance of gravitational scattering, JHEP 07 (2014) 152 [arXiv: 1312.2229] [INSPIRE].

[4] T. He, V. Lysov, P. Mitra and A. Strominger, BMS supertranslations and Weinberg's soft graviton theorem, arXiv:1401.7026 [INSPIRE].

[5] D. Kapec, V. Lysov, S. Pasterski and A. Strominger, Semiclassical Virasoro symmetry of the quantum gravity $\mathcal{S}$-matrix, JHEP 08 (2014) 058 [arXiv:1406.3312] [INSPIRE].

[6] H. Bondi, M. van der Burg and A. Metzner, Gravitational waves in general relativity. $\%$ Waves from axisymmetric isolated systems, Proc. Roy. Soc. Lond. A 269 (1962) 21.

[7] R. Sachs, Gravitational waves in general relativity. 8. Waves in asymptotically flat space-times, Proc. Roy. Soc. Lond. A 270 (1962) 103.

[8] F. Cachazo and A. Strominger, Evidence for a new soft graviton theorem, arXiv:1404.4091 [INSPIRE].

[9] F.E. Low, Bremsstrahlung of very low-energy quanta in elementary particle collisions, Phys. Rev. 110 (1958) 974 [INSPIRE].

[10] T.H. Burnett and N.M. Kroll, Extension of the low soft photon theorem, Phys. Rev. Lett. 20 (1968) 86 [INSPIRE].

[11] J.S. Bell and R. Van Royen, On the low-Burnett-Kroll theorem for soft-photon emission, Nuovo Cim. A 60 (1969) 62 [inSPIRE].

[12] V. Del Duca, High-energy bremsstrahlung theorems for soft photons, Nucl. Phys. B 345 (1990) 369 [inSPIRE].

[13] D.J. Gross and R. Jackiw, Low-energy theorem for graviton scattering, Phys. Rev. 166 (1968) 1287 [INSPIRE].

[14] C.D. White, Factorization properties of soft graviton amplitudes, JHEP 05 (2011) 060 [arXiv:1103.2981] [INSPIRE].

[15] E. Casali, Soft sub-leading divergences in Yang-Mills amplitudes, arXiv:1404.5551 [INSPIRE].

[16] A.J. Larkoski, Conformal invariance of the subleading soft theorem in gauge theory, Phys. Rev. D 90 (2014) 087701 [arXiv:1405.2346] [INSPIRE].

[17] J. Broedel, M. de Leeuw, J. Plefka and M. Rosso, Constraining subleading soft gluon and graviton theorems, Phys. Rev. D 90 (2014) 065024 [arXiv:1406.6574] [INSPIRE].

[18] C.D. White, Diagrammatic insights into next-to-soft corrections, Phys. Lett. B 737 (2014) 216 [arXiv:1406.7184] [inSPIRE].

[19] Z. Bern, S. Davies, P. Di Vecchia and J. Nohle, Low-energy behavior of gluons and gravitons from gauge invariance, Phys. Rev. D 90 (2014) 084035 [arXiv:1406.6987] [INSPIRE].

[20] F. Cachazo, S. He and E.Y. Yuan, Scattering of massless particles in arbitrary dimensions, Phys. Rev. Lett. 113 (2014) 171601 [arXiv:1307.2199] [INSPIRE].

[21] B.U.W. Schwab and A. Volovich, Subleading soft theorem in arbitrary dimensions from scattering equations, Phys. Rev. Lett. 113 (2014) 101601 [arXiv: 1404.7749] [INSPIRE].

[22] N. Afkhami-Jeddi, Soft graviton theorem in arbitrary dimensions, arXiv:1405.3533 [INSPIRE]. 
[23] Z. Bern, S. Davies and J. Nohle, On loop corrections to subleading soft behavior of gluons and gravitons, Phys. Rev. D 90 (2014) 085015 [arXiv: 1405.1015] [INSPIRE].

[24] S. He, Y.-t. Huang and C. Wen, Loop corrections to soft theorems in gauge theories and gravity, JHEP 12 (2014) 115 [arXiv: 1405.1410] [INSPIRE].

[25] F. Cachazo and E.Y. Yuan, Are soft theorems renormalized?, arXiv:1405.3413 [INSPIRE].

[26] B.U.W. Schwab, Subleading soft factor for string disk amplitudes, JHEP 08 (2014) 062 [arXiv: 1406.4172] [INSPIRE].

[27] M. Bianchi, S. He, Y.-t. Huang and C. Wen, More on soft theorems: trees, loops and strings, arXiv: 1406.5155 [INSPIRE].

[28] T. Adamo, E. Casali and D. Skinner, Perturbative gravity at null infinity, Class. Quant. Grav. 31 (2014) 225008 [arXiv:1405.5122] [INSPIRE].

[29] Y. Geyer, A.E. Lipstein and L. Mason, Ambitwistor strings at null infinity and subleading soft limits, arXiv:1406.1462 [INSPIRE].

[30] T. He, P. Mitra, A.P. Porfyriadis and A. Strominger, New symmetries of massless QED, JHEP 10 (2014) 112 [arXiv:1407.3789] [INSPIRE].

[31] V. Lysov, S. Pasterski and A. Strominger, Low's subleading soft theorem as a symmetry of QED, Phys. Rev. Lett. 113 (2014) 111601 [arXiv:1407.3814] [InSPIRE].

[32] M. Zlotnikov, Sub-sub-leading soft-graviton theorem in arbitrary dimension, JHEP 10 (2014) 148 [arXiv:1407.5936] [INSPIRE]. 